\title{
Influence of Real-Fluid Properties in Modeling Decompression Wave Interacting with Ductile Fracture Propagation
}

\author{
A. Terenzi ${ }^{1}$ \\ 1 Snamprogetti SpA, Field Upstream Facilities and Pipelines Division, via Toniolo n. 1, 61032 Fano (PU) - Italy \\ e-mail: alessandro.terenzi@snamprogetti.eni.it
}

\begin{abstract}
Résumé - Influence des propriétés du fluide réel dans la modélisation de l'onde de détente interférant avec la propagation de la fracture ductile — La force à l'origine de la propagation de la fracture ductile dans un conduit contenant un fluide compressible est déteminée par la pression résiduelle du fluide (qui agit contre les parois internes) tandis qu'il s'écoule à l'extérieur. L'intensité de la force est définie par le profil de pression de l'onde de détente qui se propage dans le conduit. Le traitement classique de ce phénomène est fondé sur l'hypothèse du gaz parfait et de la similarité des écoulements, avec conservation de l'entropie. Dans cet article, on considère une équation d'état des fluides réels d'hydrocarbures, avec une attention particulière sur les conditions d'écoulement diphasique en régime gaz-condensat, sous l'hypothèse d'un modèle homogène en équilibre thermodynamique. Les propriétés du fluide réel influencent la propagation de l'onde de détente via des phénomènes de condensation et de vaporisation, la vitesse du son et des effets de friction. Une approche thermodynamique et mécanique des fluides de ce problème est ici présentée, ainsi que des résultats de simulation issus d'applications industrielles.
\end{abstract}

\begin{abstract}
Influence of Real-Fluid Properties in Modeling Rarefaction Wave Interacting with Ductile Fracture Propagation - The force causing the ductile fracture propagation in pipelines containing pressurized compressible fluids is given by the residual pressure of the discharging fluid, acting on the internal wall downstream of the crack tip, in the opening flap area. The value of this force is determined by the pressure profile of the rarefaction wave propagating in the pipeline. The classical treatment of this phenomenon is based on the assumption of perfect gas behaviour and similarity flow, in which conservation of entropy holds. In this context real hydrocarbon fluids are considered, and simulated by using real EOS with special focusing on two-phase flow conditions in the gas-condensate regime, assuming a homogeneous thermodynamic equilibrium model. Real-fluid properties influence the decompression wave propagation, through condensation/vaporization phenomena, sound velocity trend and frictional effects. A treatment of the thermodynamics and fluid mechanics of this problem is presented, as well as simulation results relevant to industrial applications.
\end{abstract}




\section{INTRODUCTION}

The propagating shear fracture analysis is an important step for the fracture control of natural gas transmission pipelines. The phenomenon consists in the interaction between the fluid decompression and the crack propagation. When the crack has been generated, the internal fluid escapes from the ruptured portion of the pipeline and the rarefaction wave proceeds toward the pipeline ends $[1,2]$. The crack evolution depends on the rarefaction wave velocity, since the crack front propagation may be stopped by a low fluid residual pressure, in a flow region where the decompression has already passed. The simpler description of the decompression wave is obtained by assuming one-dimensional isentropic homogeneous equilibrium flow; in case of absence of condensation, a full analytical approach is possible for a perfect gas model. The assumption of one-dimensional flow through a full-bore opening is valid except for a short period when the flaps behind the crack tip are not yet fully opened. In the following, the approximation of isentropic flow is left, because the influence of wall friction is taken into account; however the adiabaticity is kept since the phenomena under consideration are so fast that the heat transfer effects have no enough time to manifest. Real fluid thermodynamic properties expressed by using appropriate EOS (Equation Of State) are taken into account as well as possible liquid condensation. If two-phase flow occurs, it is considered as homogeneous and in thermodynamic equilibrium. The assumption of homogeneity is justified by the high velocity of flow in the wave, where probably liquid droplets are transported by the gas core. Equilibrium conditions are present when the high velocity system is supplied by a stream having large void fraction $[3,4]$. In fact the transport systems here considered usually contain gas only or gas with small amount of liquid ("gas-condensate" fluids).

In the following, the description of the expansion wave model based on the perfect gas assumption is presented at first; then the real fluid calculation scheme is discussed and its implementation into a new simulation tool is presented. Some simulations of pipeline ruptures are shown; the first is referred to burst tests carried out in Canada, in which calculation results are compared to experimental data. In this case the fluid in the wave is gas with a very small amount of liquid. The second simulation is concerned with a recently designed pipeline, transporting a fluid in a two-phase stratified flow regime, with a liquid content significantly higher than the previous one. Both calculations allow to evaluate the difference between the perfect gas and real fluid approaches, as well as the influence of effects as wall friction and liquid condensation.

\section{PERFECT GAS APPROACH}

The generation of the rarefaction wave inside a gas pipeline following a rupture may be treated as a similarity flow in a perfect gas [5]. This means that the distributions of all quantities can depend on the coordinate $x$ and the time $t$ only through the ratio $x / t$, which has the dimensions of velocity. Therefore, these distributions at various instants will be similar, differing only in the scale along the $x$-axis, which increases proportionally to the time. We can say that, if lengths are measured in a unit which increases proportionally to $t$, then the flow pattern does not change. Hence the local values of fluid velocity $v$, sound velocity $c$ and pressure $p$ as functions of stagnation values in the undisturbed pressurized gas (indicated by the suffix 0 ) are:

$$
\begin{gathered}
|v|=\frac{2}{\gamma+1}\left(c_{0}-\frac{x}{t}\right) \\
c=c_{0}-\frac{\gamma-1}{2}|v| \\
p=p_{0}\left[1-\frac{\gamma-1}{\gamma+1}\left(1-\frac{v_{e}}{c_{0}}\right)\right]^{\frac{2 \gamma}{\gamma-1}}
\end{gathered}
$$

where $\gamma$ is the isentropic coefficient.

The local pressure $p$ has been expressed as a function of the local wave expansion velocity $v_{e}$, given by the difference between the local sound and fluid velocities $\left(v_{e}=c-|v|\right)$. The above equations have been derived by coupling the condition of constant entropy to the equations of continuity and the $x$-component of Euler's equation, by neglecting friction and heat transfer [5].

In Figures 1 and 2 the velocities and pressure ratio $\left(=p / p_{0}\right)$ profiles along a decompression wave in a perfect gas having $c_{0}=400 \mathrm{~m} / \mathrm{s}$ and $\gamma=1.3$ are shown. The curve shown in Figure 1 is called "decompression curve". From Figure 2 it is evident that the rarefaction wave is bounded on one side by a region where the gas is at rest, and where the sound velocity has its maximum value, giving the propagation velocity of the wave front. Along the wave the fluid velocity increases and the sound velocity decreases, due to the decompression effect, until the two values coincide at the rupture location, where the expansion is locked. The exit pressure ratio, i.e. the ratio between the pressure at the rupture location $p_{\text {exit }}$ and the stagnation pressure $p_{0}$, has the following expression:

$$
\frac{p_{\text {exit }}}{p_{0}}=\left(\frac{2}{\gamma+1}\right)^{\frac{2 \gamma}{\gamma-1}}
$$

which is about 0.3 for $\gamma=1.3,1.4$.

\section{REAL FLUID APPROACH}

In the oil and gas industry, thermodynamic properties of hydrocarbon mixtures are usually derived by applying real fluid EOS; the more accurate predictions are given by SRK (Soave-Redlich-Kwong, [6]) and PR (Peng-Robinson, [7]). 


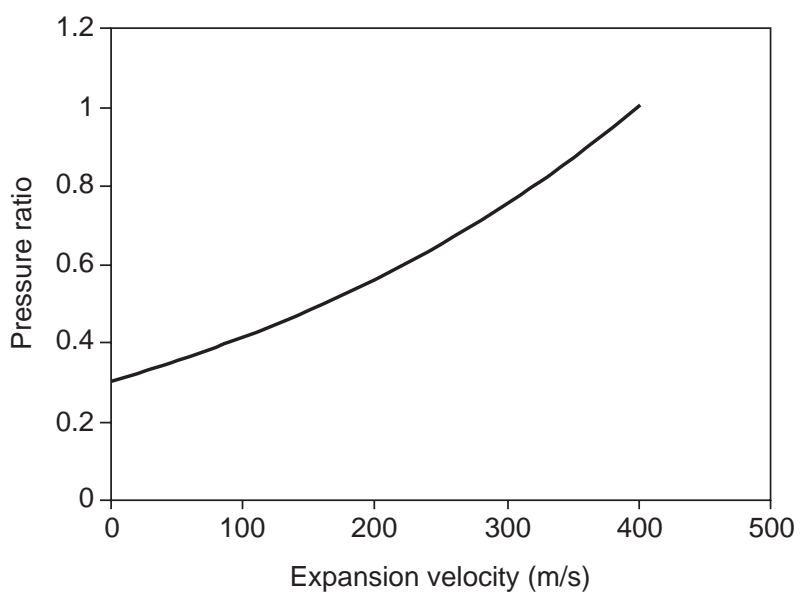

Figure 1

Pressure ratio in a perfect gas decompression curve.

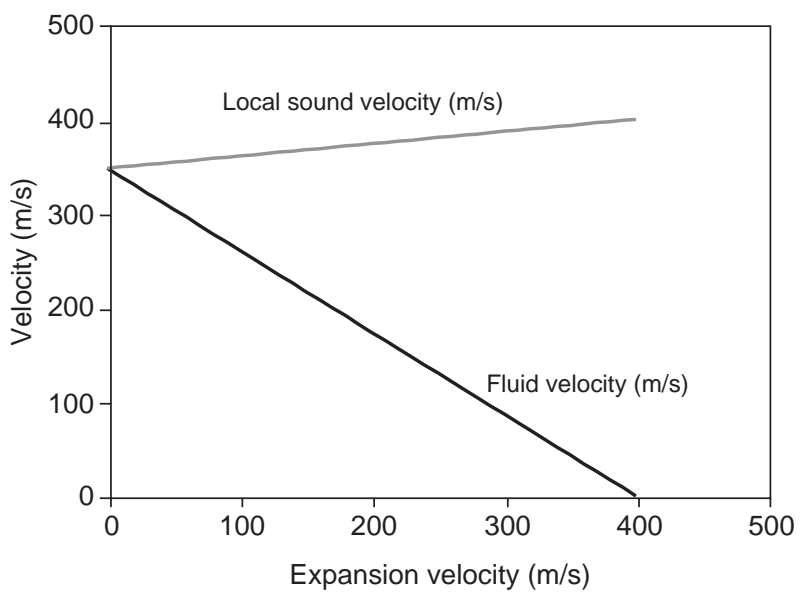

Figure 2

Fluid and sound velocities in a perfect gas decompression curve.
These equations are modifications of the classical cubic EOS of Van der Waals; their applications does not allow an analytical treatment similar to that of the perfect gas model. However, to have an idea of the possible expressions of flow parameters, let us define the isentropic $\gamma$ and Gruneisen $\Gamma$ coefficients for a real gas $[4,8,9]$ :

$$
\begin{gathered}
\gamma=\frac{\rho}{p} \frac{C_{P}}{C_{V}} \frac{1}{\left(\frac{\partial \rho}{\partial P}\right)_{T}} \\
\Gamma=\frac{h}{e}-1
\end{gathered}
$$

where $\rho$ is the gas density, $C_{P}$ and $C_{V}$ are the gas heat capacities at constant pressure and constant volume respectively, $T$ is the temperature, $h$ and $e$ are the gas specific enthalpy and internal energy. Assuming that $\gamma, \Gamma$ are constant along the rarefaction wave thermodynamic path (considered as isentropic; actually for a real gas they are constant in a local sense only), the relations between the thermodynamic variables in the wave are:

$$
\begin{aligned}
\frac{p}{p_{0}} & =\left(\frac{\rho}{\rho_{0}}\right)^{\gamma} \\
\frac{T}{T_{0}} & =\left(\frac{\rho}{\rho_{0}}\right)^{\Gamma} \\
\frac{T}{T_{0}} & =\left(\frac{p}{p_{0}}\right)^{\Gamma / \gamma} \\
\frac{p_{\text {exit }}}{p_{0}} & =\left(\frac{2}{\gamma+1}\right)^{\frac{2 \gamma}{\gamma-1}}
\end{aligned}
$$

If the stagnation fluid was pure methane at $15 \mathrm{MPa}$ and $0^{\circ} \mathrm{C}$, the above exit pressure ratio would be 0.192 , since $\gamma=2.055$.

In case of presence of a liquid phase, considered as formed by condensation caused by the fluid cooling associated to the expansion or as already existing in the initial condition, with the assumption of homogeneity the fluid can be thought as having uniform averaged properties and can be treated as a perfect mixture. The density of the homogeneous gas-liquid mixture $\rho_{T W O}$ may be expressed by [4]:

$$
\rho_{T W O}=\alpha \rho_{G}+(1-\alpha) \rho_{L}
$$

where $\rho_{G}$ and $\rho_{L}$ are the gas and liquid densities, and $\alpha$ is the void fraction.

For this kind of mixtures the sound velocity is a sort of weighted average between the single phases sound velocities [10]:

$$
\frac{1}{c_{T W O}^{2}}=\rho_{T W O}\left(\frac{\alpha}{\rho_{G} c_{G}^{2}}+\frac{1-\alpha}{\rho_{L} c_{L}^{2}}\right)
$$

where $c_{T W O}$ is the gas-liquid two-phase mixture sound velocity, $c_{G}$ and $c_{L}$ are the single phase gas and liquid sound velocities.

The comparison of Equation (12) with experiments is shown in Figure 3, drawn by [10].

It becomes apparent that in the two-phase regime, the sound propagation velocity may be much lower than the smallest one of the two single phases, up to one order of magnitude; this means that the two-phase mixture appears to be much more compressible than the gas. The physical interpretation of this behaviour is based on the interaction of the wave with the elastic boundaries represented by the interfaces between phases, giving a celerity decrease similar 
to that of a pressure pulse propagating in a pipe with elastic walls [11].

A comprehensive simulation tool (Machnet) of fast transient flow of compressible fluids in pipelines, taking care of real fluid thermodynamic properties, friction and heat transfer phenomena, based on a finite volume integration of the whole set of flow conservation equations has been developed [4, 12].

The one-dimensional conservative form of the flow equations is used [13]:

$$
\begin{gathered}
\frac{\partial \rho}{\partial t}+\frac{\partial(\rho v)}{\partial x}=-\frac{1}{S} \frac{d S}{d x} \rho v \\
\frac{\partial(\rho v)}{\partial t}+\frac{\partial\left(\rho v^{2}+p\right)}{\partial x}=-\frac{1}{S} \frac{d S}{d x} \rho v^{2}+\tau \\
\frac{\partial(\rho E)}{\partial t}+\frac{\partial(\rho v H)}{\partial x}=-\frac{1}{S} \frac{d S}{d x} \rho v H+q_{H}
\end{gathered}
$$

where $S$ is the pipe internal cross section, $E$ and $H$ are the total energy and enthalpy per unit mass, $\tau$ and $q_{H}$ are the source terms due to friction and thermal exchange between gas and pipe wall, given by:

$$
\begin{gathered}
\tau=-\frac{f}{R_{P}} \rho v^{2} \\
q_{H}=-\hat{h}_{i}\left(T_{a w}-T_{\text {wall }}\right)
\end{gathered}
$$

where $f$ is the Fanning friction factor, $R_{P}$ the pipe internal radius, $\hat{h}_{i}$ the internal heat transfer coefficient, $T_{a w}$ and $T_{\text {wall }}$ the adiabatic wall and internal pipe wall temperatures respectively. The adiabatic wall temperature [14] is defined on the basis of the recovery factor $\mathfrak{R}$, which is a measure of the fraction of the local free stream dynamic temperature rise recovered at the wall; it can be defined as a function of the bulk gas temperature $T$ and its specific heat at constant pressure $C_{P}$ in this way:

$$
T_{a w}=T+\frac{\Re}{2} \frac{v^{2}}{C_{P}}
$$

The heat transfer between the fluid and the external environment is calculated by considering the radial conduction process through the pipe wall layers (by solving the Fourier equation in cylindrical geometry) and the heat transfer on the external pipe surface (the environment may be ground, air or sea water).

For what concern the friction modelling, in this work the Colebrook-White correlation for the friction factor covering both smooth and rough pipes is assumed [15]:

$$
\frac{1}{\sqrt{4 f}}=-2 \log \left[\frac{\varepsilon}{3.7 D_{P}}+\frac{2.51}{\operatorname{Re} \sqrt{4 f}}\right]
$$

where $\mathrm{Re}$ is the pipe Reynolds number based on diameter $D_{P}$ and $\varepsilon$ is the pipe roughness.

The numerical method used in Machnet is known as Roe's method. It is a finite volume conservative scheme of the Godunov-type $[13,16]$, in which the approximate Riemann solver is based on a characteristic decomposition of the flux differences ensuring the conservation properties. Roe's approach extends the linear wave decomposition, which is the exact linear solution to the Riemann problem, to nonlinear equations.

In this method, the following expressions for the interfaces fluxes $F_{j+1 / 2}^{*}, F_{j-1 / 2}^{*}$ referred to the calculation cell $j$ result:

$$
\begin{aligned}
& F_{j+1 / 2}^{*}=F_{j}+\tilde{\mathbf{A}}^{-}\left(U_{j+1}-U_{j}\right) \\
& F_{j-1 / 2}^{*}=F_{j}-\tilde{\mathbf{A}}^{+}\left(U_{j}-U_{j-1}\right)
\end{aligned}
$$

Formulae (20), (21) represent a flux splitting approach typical of upwind methods [13], applying a discretization that depends on the propagation direction of the waves. In the above expressions $U_{j}$ and $F_{j}$ are the conservative variable and its flux referred to $j$-th cell, while $\tilde{\mathbf{A}}^{-}$and $\tilde{\mathbf{A}}^{+}$are the split Jacobians based on right and left eigenvectors and eigenvalues.

The weak formulation of the first order explicit upwind Roe's method for the Navier-Stokes equations, used in this work, giving the conservative state variable $U_{j}$ at time $(n+1) \Delta t$ as a function of previous value at $n \Delta t$ is:

$$
U_{j}^{n+1}-U_{j}^{n}=-\frac{\Delta t}{\Delta x}\left(F_{j+1 / 2}^{*}-F_{j-1 / 2}^{*}\right)+(\text { source term })
$$

where $\Delta t$ and $\Delta x$ are the time and space discretization steps.

By solving the set of equations (22) on the calculation grid, the conservative solution vector $\vec{U}=(\rho, \rho v, \rho E)$ is known at a specified time step. The other thermodynamic properties, as for instance the void fraction, are obtained by linear interpolation through a table of values having the couple $(\rho, \rho e)$ as independent variables. This table is created in advance by inverting a standard PVT thermodynamic table (i.e. having pressure and temperature as independent variables), supplied by commercial hydrocarbon PVT codes like PVTsim (by Calsep, [17]), in the considered pressure and temperature calculation range. Since the assumption of homogeneous equilibrium is applied, the possible phase change is considered as instantaneous, in such a way vapour and liquid are always in thermodynamic equilibrium at the local pressure and temperature conditions.

\section{SIMULATIONS}

The simulation tool Machnet described above has been applied to cases of industrial interest. The first set of simulations was carried out in order to compare Machnet predictions with experimental measurements. In [2] burst 


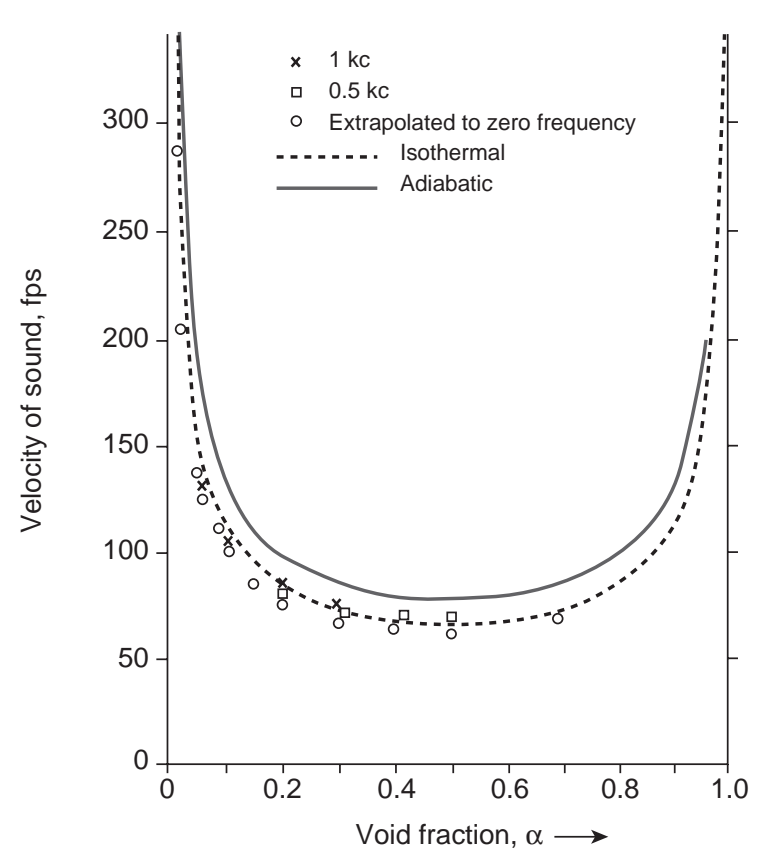

Figure 3

Sound velocity versus void fraction in a two-phase homogeneous mixture (from Wallis [10]).

tests conducted by a Canadian Company (Foothills Pipelines) at the Northern Alberta Burst Test Facility in 1981, in order to study the process of ductile fracture propagation in pipelines, are reported. The fluid mixtures under consideration (natural gas with about $85 \%$ of methane) were described by using real fluid EOS (SRK and PR), and condensation effects occurred during the tests. Actually the two EOS give very similar predictions of fluid thermodynamic properties in this case (see Appendix A). Pipes diameters were selected in the range $1219 / 1422 \mathrm{~mm}$, pressures in the range $7.5 / 8.7 \mathrm{MPa}$, temperatures in the range $-18 /+18^{\circ} \mathrm{C}$.

Experimental data are referred to the expansion wave generated by burst and propagated inside the pipeline. They show the pressure ratio for each point of the wave $v s$. the expansion velocity $v_{e}$. In this case the flow may be considered isentropic, considering that the lengths of pipes used in the tests were short (of the order of $60 \mathrm{~m}$ ). Hence simulations were carried out by neglecting friction and heat transfer effects. One test has been selected among the ones reported in [2], namely NABT Test no. 5. In this case the pipeline diameter was $1422 \mathrm{~mm}$, the initial pressure was 7.544 MPa, the initial temperature was $18.5^{\circ} \mathrm{C}$. Table 1 shows the relevant gas molar composition. In Figure 4 a comparison between Machnet results and experiments is shown. The void fraction profile in the expansion wave is shown along with the pressure ratio; in this case gas only was present in the pipeline initial condition before rupture, and some liquid was formed during the expansion wave
TABLE 1

Fluid compositions used in pipeline rupture simulations

\begin{tabular}{l|c|c}
\hline Component & NABT Test no. 5 & $\begin{array}{c}711 \text { mm gas-condensate } \\
\text { pipeline }\end{array}$ \\
\hline $\mathrm{N} 2$ & 2.212 & 1.134 \\
$\mathrm{CO}_{2}$ & 0 & 5.046 \\
$\mathrm{H}_{2} \mathrm{~S}$ & 0 & 16.987 \\
$\mathrm{C}_{1}$ & 84.694 & 60.513 \\
$\mathrm{C}_{2}$ & 8.212 & 8.802 \\
$\mathrm{C}_{3}$ & 4.379 & 4.285 \\
$i-\mathrm{C}_{4}$ & 0.201 & 0.650 \\
$n-\mathrm{C}_{4}$ & 0.235 & 1.299 \\
$i-\mathrm{C}_{5}$ & 0.029 & 0.360 \\
$n-\mathrm{C}_{5}$ & 0.030 & 0.358 \\
$n-\mathrm{C}_{6}$ & 0.008 & 0.289 \\
$n-\mathrm{C}_{7}$ & 0 & 0.146 \\
$n-\mathrm{C}_{8}$ & 0 & 0.071 \\
$n-\mathrm{C}_{9}$ & 0 & 0.019 \\
$n-\mathrm{C}_{10}$ & 0 & 0.010 \\
$n-\mathrm{C}_{11}$ & 0 & 0.004 \\
$n-\mathrm{C}_{12}$ & 0 & 0.002 \\
$n-\mathrm{C}_{13}$ & 0 & 0.001 \\
Benzene $_{\text {Toluene }}$ & 0 & 0.006 \\
$p-x y l e n e$ & 0 & 0.008 \\
& 0 & 0.006 \\
\hline
\end{tabular}

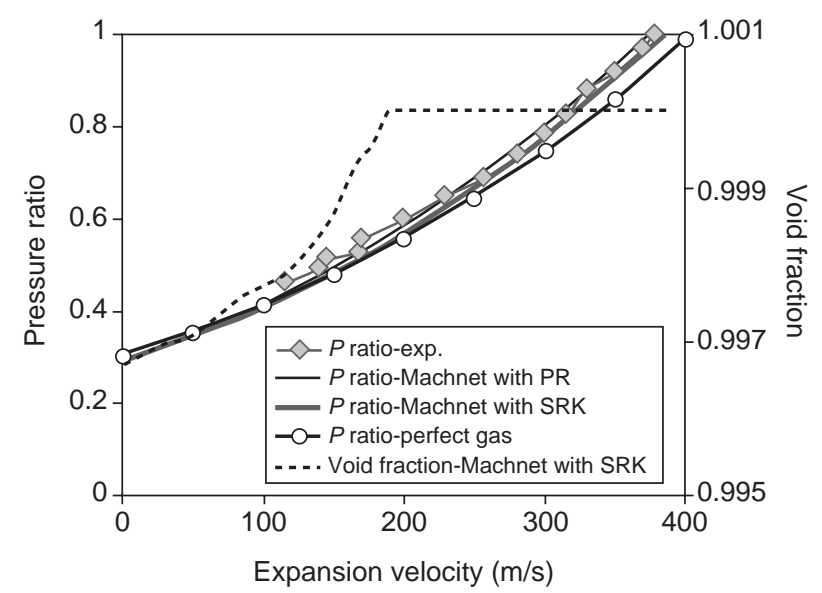

Figure 4

NABT Test no. 5 decompression curve.

propagation. Figure 4 shows the expansion wave calculated with the perfect gas assumption also (see Eq. (3)). An estimation of the difference between perfect gas and real fluid models, about the expansion wave pressure prediction, can be given for a selected value of expansion velocity; for instance, by considering a velocity of $350 \mathrm{~m} / \mathrm{s}$ the real fluid model predicts $7.02 \mathrm{MPa}$, and the perfect gas $6.49 \mathrm{MPa}$, with a difference of $8 \%$. Figure 5 shows the thermodynamic path followed by the fluid along the expansion wave, along with 


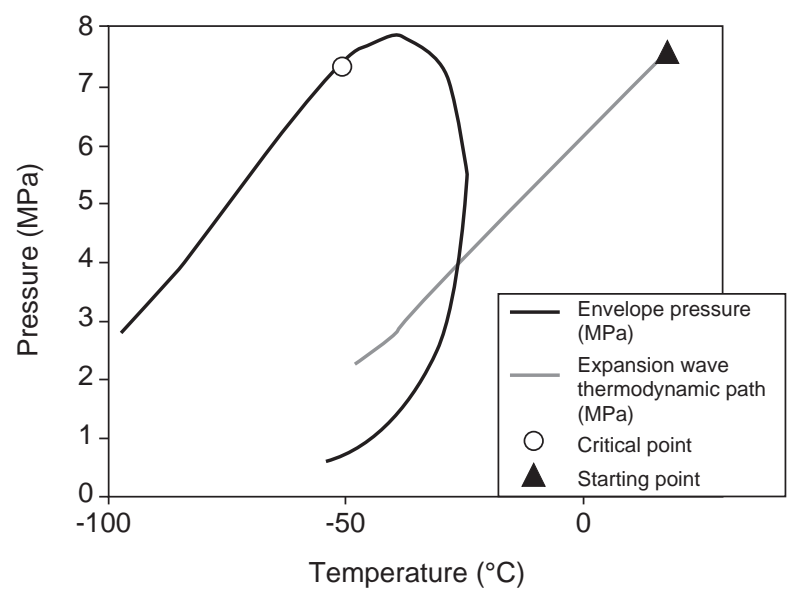

Figure 5

Expansion wave thermodynamic path in the NABT Test no. 5 referred to the phase envelope diagram.

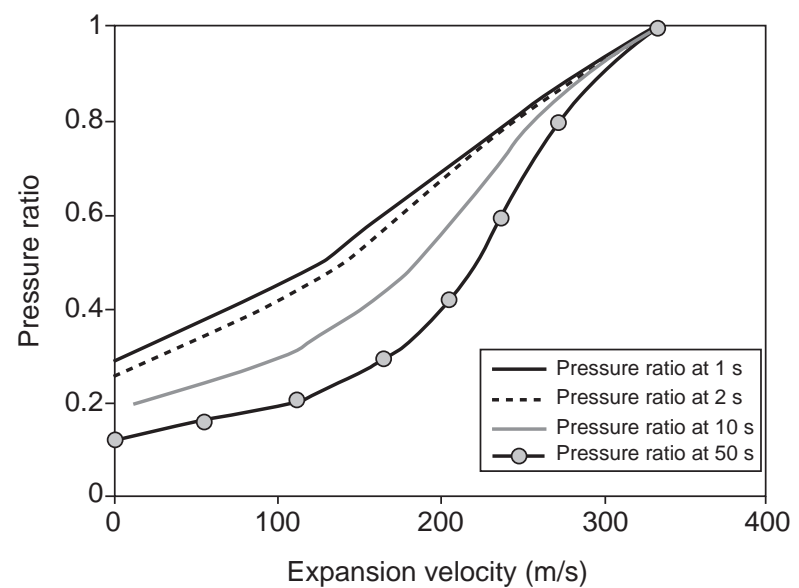

Figure 6

Pressure ratio in the $711 \mathrm{~mm}$ gas-condensate pipeline decompression curve. the phase envelope curve in a $p-T$ diagram; it is evident that the initial condition of the undisturbed fluid is in the overheated gas region, and the pressure reduction brings the fluid inside the two-phase region causing liquid condensation. A good agreement can be observed between program predictions and experiments.

A further simulation has been carried out for a new pipeline, designed to transport a two-phase fluid (gascondensate) in the Kazakhstan area. The pipeline has a nominal diameter of $711 \mathrm{~mm}$, length $100 \mathrm{~km}$, and the methane molar percent in the fluid composition is about $60 \%$. The whole molar composition of this fluid is reported in Table 1 . Thermodynamic properties has been calculated by the SRK EOS. The initial condition for the rupture simulation is with the pipeline pressurized at $9.86 \mathrm{MPa}, 7^{\circ} \mathrm{C}$ and a void fraction of 0.886 . For this configuration, the simulation has been carried out by including friction. Furthermore the initial condition is referred to a two-phase fluid, in static conditions; this means that actually the flow pattern is stratified at the rarefaction wave front location.

Figure 6 shows the shape of the expansion wave at different times from the rupture instant. It is evident the lowering of the curves with time, which is caused by the wall friction (in opposition to isentropic flow where a single expansion curve is defined valid for all times, since it is a similarity flow).

Figure 7 shows the void fraction profiles in the expansion wave at different times; in this case, unlike the NABT test, some liquid is evaporated due to the pressure decrease caused by the wave.

Figure 8 shows the thermodynamic path followed by the expanding fluid in this case (referred to $1 \mathrm{~s}$ after the rupture); the initial condition is located inside the two-phase region and the expansion brings the fluid toward lower $p-T$ states inside this region, where the pressure drop effect prevails on the temperature reduction giving thermodynamic conditions with higher vapour content.

Figure 9 shows the sound velocity profiles in the expansion wave at different times; it is interesting to observe that at later times there is a balancing conflict between the evaporation effect which would give an increase in sound velocity and depressurization which would give a decrease; this produces a sort of plateau in the middle of the wave.

A check must be carried out to evaluate the approximation of considering the mixture sound velocity as given by the homogenous flow formula in the whole flow field, Equation (12), and the following expression for stratified flow, drawn by [11]:

$$
\begin{gathered}
c_{G, s}^{2}=\frac{1}{\frac{1}{c_{G}^{2}}+\frac{1-\alpha}{\alpha} \frac{\rho_{G}}{\rho_{L}} \frac{1}{c_{L}^{2}}} \\
c_{L, s}^{2}=\frac{1}{\frac{1}{c_{L}^{2}}+\frac{\alpha}{1-\alpha} \frac{\rho_{L}}{\rho_{G}} \frac{1}{c_{G}^{2}}}
\end{gathered}
$$

where $c_{G, s}$ and $c_{L, s}$ are the sound velocities in the gas and liquid phases for stratified flow conditions.

As stated in [11], for stratified one-dimensional flow a composite sonic velocity does not exist, because each of the separated phases is continuous in axial direction. If a pressure pulse is imposed on the liquid and gas at the same time, the disturbance propagates with different velocities in both phases in axial direction (parallel to the interface). However, due to the higher compressibility of the gas phase, the local pressure is mainly determined by it, and a comparison must be carried out between (23) and (12) predictions. By using 


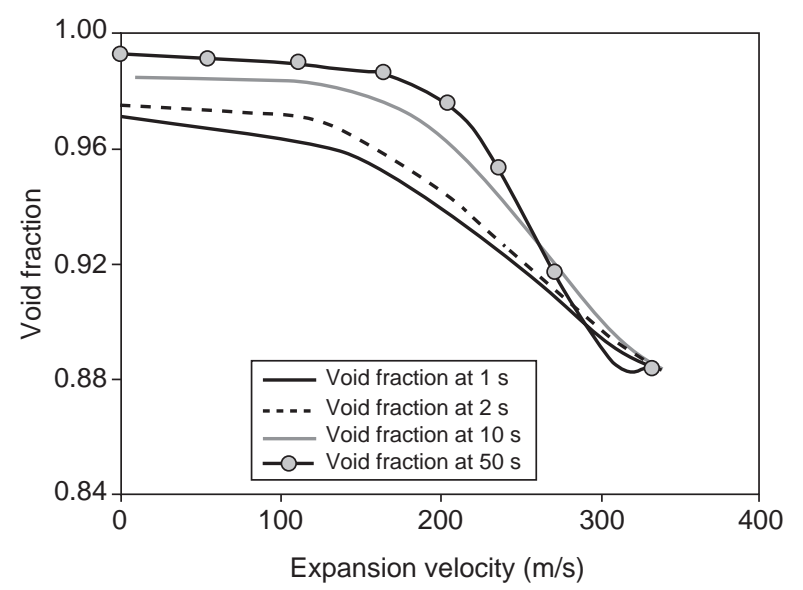

Figure 7

Void fraction in the $711 \mathrm{~mm}$ gas-condensate pipeline decompression curve.

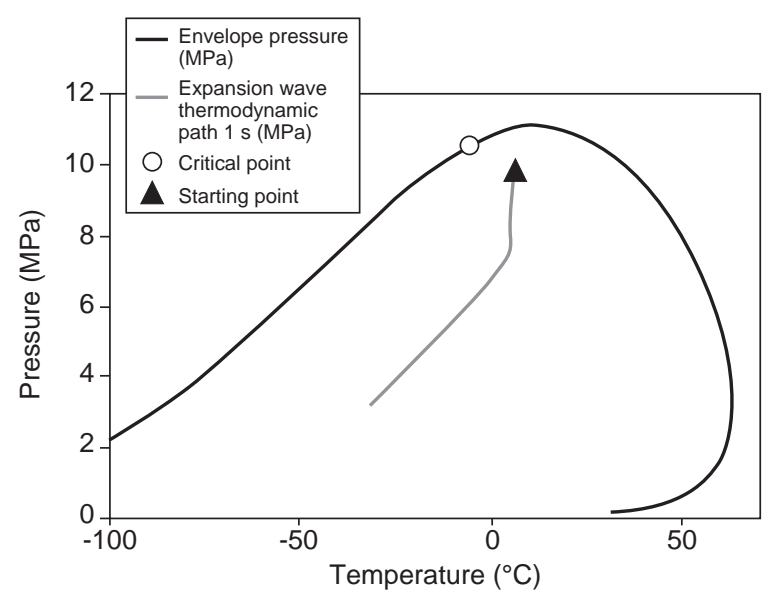

Figure 8

Expansion wave thermodynamic path in the $711 \mathrm{~mm}$ gascondensate pipeline referred to the phase envelope diagram.

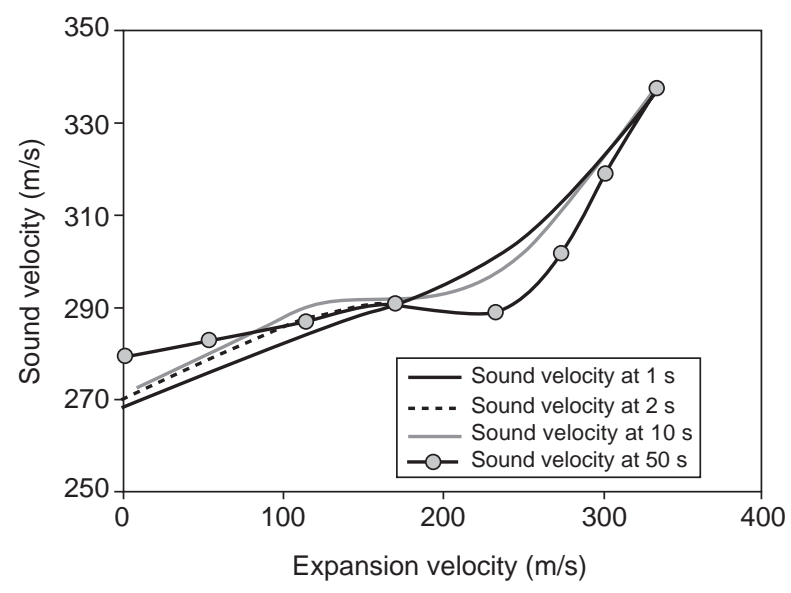

Figure 9

Sound velocity in the $711 \mathrm{~mm}$ gas-condensate pipeline decompression curve. the PR EOS for the subject fluid composition, a flash at initial conditions gives the following values:

$$
\begin{gathered}
\rho_{G}=186.4 \mathrm{~kg} / \mathrm{m}^{3} \\
\rho_{L}=394.5 \mathrm{~kg} / \mathrm{m}^{3} \\
c_{G}=342.3 \mathrm{~m} / \mathrm{s} \\
c_{L}=481.6 \mathrm{~m} / \mathrm{s}
\end{gathered}
$$

The following values are obtained for the average density and sound velocities:

$$
\begin{gathered}
\rho_{T W O}=210 \mathrm{~kg} / \mathrm{m}^{3} \\
c_{T W O}=337 \mathrm{~m} / \mathrm{s} \\
c_{G, s}=337 \mathrm{~m} / \mathrm{s} \\
c_{L, s}=83 \mathrm{~m} / \mathrm{s}
\end{gathered}
$$

Thus in this particular case, the homogeneous two-phase and stratified gas sound velocities coincide. By comparing (12) and (23), it may be concluded that this condition is caused by the following equality:

$$
\rho_{T W O} \approx \frac{\rho_{G}}{\alpha}
$$

that may be realized when:

$$
\frac{\rho_{L}}{\rho_{G}}=\frac{1+\alpha}{\alpha}(\approx 2 \text { for } \alpha \text { close to } 1)
$$

Figure 10 shows a comparison between the decompression curve calculated in isentropic flow and for the current friction case at the first instants of propagation. The curves are almost superimposed, confirming the analysis made for the previous NABT test. In this case also an expansion wave calculated with the perfect gas model is drawn in order to visualize the difference with the real fluid model; for an expansion velocity of $250 \mathrm{~m} / \mathrm{s}$, the real fluid model predicts an expansion wave pressure of $8.04 \mathrm{MPa}$, while the perfect gas model gives $7.22 \mathrm{MPa}$, with a difference of $11 \%$. Obviously in the current test the influence of friction is greater since the pipe diameter is smaller, and it is more pronounced when the wave has propagated for long distances, involving a significant pipe length in flow conditions. Therefore, the two real-fluid effects considered above, i.e. friction and twophase conditions, have two opposite consequences on the decompression curve:

- friction causes a pressure reduction, i.e. it hinders the crack propagation;

- two-phase flow causes a sound velocity decrease with respect to single phase conditions, i.e. it favours the crack propagation.

However, from a design point of view, since the decompression curve must be compared with the crack propagation curves based on Battelle theory [1], and the interaction must be studied in the first instants of the propagation when the crack must be arrested by using 


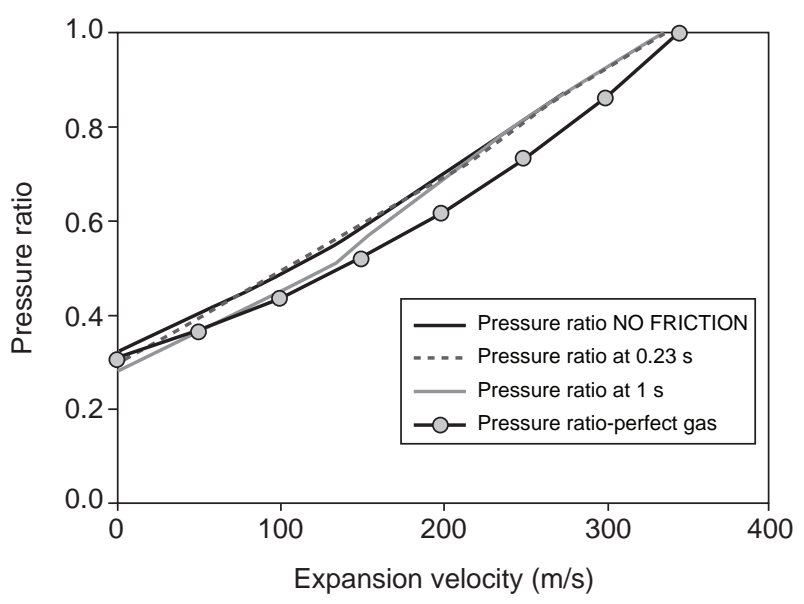

Figure 10

Pressure ratio in the $711 \mathrm{~mm}$ gas-condensate pipeline decompression curve: comparison between a frictionless calculation and the early curves of the friction calculation.

suitable devices (crack arrestors), the most conservative formulation of isentropic flow, without the friction mitigation influence, should be used.

\section{CONCLUSION}

It is a crucial point to take care of real fluid behaviour when analyzing the decompression properties of dense natural gas mixtures. The assumption of perfect gas may give rise to significant errors. In this paper the effects of friction and liquid condensation have been studied with reference to a practical design configuration. They have two opposite consequences: the friction lowers the wave pressure level (i.e. it hinders the crack propagation), while the condensation decreases the sound velocity values (i.e. it promotes the crack propagation since it reduces the decompression wave propagation velocity). If the decompression wave calculation must be carried out during a pipeline design activity, in order to evaluate the required crack arrest energy and the possible use of crack arrestors, the use of the isentropic approximation, giving a more conservative assessment of the phenomenon, is suggested.

\section{REFERENCES}

1 Makino, H., Inoue, T., Endo, S., Kubo, T. and Matsumoto, T. (2003) Simulation Method for Crack Propagation and Arrest of Shear Fracture in Natural Gas Transmission Pipelines. 13th International Offshore and Polar Engineering Conference, Honolulu, Hawaii, USA, May 25-30.

2 Picard, D.J. and Bishnoi, P.R. (1988) The Importance of Real-Fluid Behaviour and Non-Isentropic Effects in Modelling Decompression Characteristics of Pipeline Fluids for Application in Ductile Fracture Propagation Analysis. The Canadian Journal of Chem. Eng., 66, 3-12.

3 Chisholm, D. (1983) Two-Phase Flow in Pipelines and Heat Exchangers, George Godwin Ed., London, UK

4 Terenzi, A. (2001) Fast Transient Flow in Pipelines of GasCondensate Fluids. 4th International Conference on Multiphase Flow ICMF2001, New Orleans, LA, USA, May 27 to June 1.

5 Landau, L.D. and Lifshitz, E.M. (1987) Fluid Mechanics 2nd Edition, Pergamon Press, Oxford, UK.

6 Soave, G. (1972) Equilibrium Constants From a Modified Redlich-Kwong Equation of State. Chem. Eng. Sci., 27, 1197-1203.

7 Peng, D. and Robinson, D.B. (1976) A New Two-Constant Equation of State. Ind. Eng. Chem. Fundam., 15, 1, 59-64.

8 Liou, M.S., Van Leer, B. and Shuen, J.S. (1988) Splitting of Inviscid Fluxes for Real Gases. NASA Technical Memorandum 100856.

9 Saurel, R., Larini, L. and Loraud, J.C. (1994) Exact and Approximate Riemann Solvers for Real Gases. Journal of Comp. Phys., 112, 126-137.

10 Wallis, G.B. (1969) One-dimensional Two-phase Flow, Mc Graw- Hill, New York, USA.

11 Nguyen, D.L., Winter, E.R.F. and Greiner, M. (1981) Sonic Velocity in Two-Phase Systems. Int. Journal of Multiphase Flow, 7, 311-320.

12 Terenzi, A., Carniani, E., Donati, E. and Ercolani, D. (2000) Problems of Nonlinear Fluid Dynamics in Industrial Plants. In: Complex Flows in Industrial Processes, Birkhauser, Boston, USA.

13 Hirsch, C. (1990) Numerical Computation of Internal and External Flows, John Wiley \& Sons, New York, USA.

14 Shapiro, A.H. (1953) The Dynamics and Thermodynamics of Compressible Fluid Flow, John Wiley \& Sons, New York, USA.

15 Keenan, J.H. and Neumann, E.P. (1946) Measurements of Friction in a Pipe for Subsonic and Supersonic Flow of air. J. Appl. Mech., 13, A91.

16 Godunov, S., Zabrodine, A., Ivanov, M., Kraiko, A. and Prokopov, G. (1979) Resolution numérique des problèmes multidimensionnels de la dynamique des gaz, éditions MIR, Moscow, Russia.

17 PVTsim ver. 13.1, PVT and Phase Behaviour Simulation System, by Calsep.

18 Reid, R., Prausnitz, J.M. and Sherwood, T.K. (1977) The Properties of Gases and Liquids 3rd Edition, McGraw-Hill Book Co., New York, USA.

Final manuscript received in February 2005 


\section{APPENDIX A}

In the hydrocarbon processing industry, cubic EOS are widely used for their simple form and their ability to reproduce hydrocarbon mixtures thermodynamic properties. The two kinds of EOS considered in this work, namely SRK and Peng-Robinson, are derived from the original RedlichKwong equation [18], which in turn is a modification of the Van der Waals equation, having this expression:

$$
p=\frac{R T}{V-b}-\frac{a T^{-0.5}}{V(V+b)}
$$

where $R$ is the universal gas constant, $V$ the molar volume, and $a, b$ two parameters having the following form for hydrocarbon mixtures:

$$
\begin{aligned}
& a=0.42748 R^{2}\left[\sum_{i} \frac{T_{c i}^{1.25}}{p_{c i}^{0.5}} x_{i}\right]^{2} \\
& b=0.08664 R\left[\sum_{i} \frac{T_{c i}}{p_{c i}} x_{i}\right]
\end{aligned}
$$

where $T_{c i}, p_{c i}$ and $x_{i}$ are the critical temperature, critical pressure and molar fraction of the $i$-th component.

The SRK EOS [6] represents a generalization of the Redlich-Kwong equation to a wider temperature range, and giving a more detailed description of vapour/liquid equilibrium.

The equation has the following form:

$$
p=\frac{R T}{V-b}-\frac{a(T)}{V(V+b)}
$$

where now the expression for the temperature function $a(T)$ and for $b$ are, for a pure component:

$$
\begin{aligned}
a(T) & =0.42748 \frac{R^{2} T_{c}^{2}}{p_{c}} \\
& {\left[1+\left(0.48+1.574 \omega-0.176 \omega^{2}\right)\left(1-\sqrt{\frac{T}{T_{c}}}\right)\right]^{2} }
\end{aligned}
$$

$$
b=0.08664 \frac{R T_{c}}{p_{c}}
$$

where $\omega$ is the acentric factor.

The mixing rules for mixtures are based on the use of binary interaction parameters $k_{i j}$ between two components $i$ and $j$, giving the following expressions for $a$ and $b$ :

$$
\begin{gathered}
a=\sum_{i} \sum_{j}\left(1-k_{i j}\right) a_{i}^{0.5} a_{j}^{0.5} x_{i} x_{j} \\
b=\sum_{i} b_{i} x_{i}
\end{gathered}
$$

A further extension of the Redlich-Kwong equation is provided by the Peng-Robinson EOS [7]. This equation gives a better prediction of the liquid phase density, and it has the following expression:

$$
p=\frac{R T}{V-b}-\frac{a(T)}{V(V+b)+b(V-b)}
$$

with the following expression for $a(T)$ and $b$ valid for a pure component:

$$
\begin{aligned}
a(T) & =0.45724 \frac{R^{2} T_{c}^{2}}{p_{c}} \\
& {\left[1+\left(0.37464+1.54226 \omega-0.26992 \omega^{2}\right)\left(1-\sqrt{\frac{T}{T_{c}}}\right)\right]^{2} }
\end{aligned}
$$

$$
b=0.0778 \frac{R T_{c}}{p_{c}}
$$

The mixing rules for mixtures are the same as for the SRK EOS.

The application of SRK or Peng-Robinson EOS to natural gas often gives very similar predictions of thermodynamic properties, as in the case of the fluids considered in this work; as a rule of thumb, it can be said that SRK is more appropriate for lighter fluids (molecular weight $=18-20 \mathrm{~kg} / \mathrm{kmol}$ ), while Peng-Robinson is more suitable for heavier gases (molecular weight $>25 \mathrm{~kg} / \mathrm{kmol}$ ). 